this gap. METHODS/STUDY POPULATION: We tested SCS patients at 3 times points: baseline (pre-surgery), at day 7 during the trial period (post-trial), and 6 months after a permanent system had been implanted. At each time point participants completed 10 minutes of eyes closed, resting electroencephalography (EEG) and self-reported their pain. EEG was collected with the ActiveTwo system and a 128-electrode cap. Patients were grouped based on the percentage change of their pain from baseline to the final visit using a median split (super responders $>$ average responders). Spectral density powerbands were extracted from resting EEG to use as input features for machine learning analyses. We used support vector machines to predict response to SCS. RESULTS/ANTICIPATED RESULTS: Baseline and post-trial EEG data predicted SCS response at 6-months with $95.56 \%$ and $100 \%$ accuracy, respectively. The gamma band had the highest performance in differentiating responders. Post-trial EEG data best differentiated the groups with feature weighted dipoles being more highly localized in sensorimotor cortex. DISCUSSION/SIGNIFICANCE OF FINDINGS: Understanding how SCS works and who it works best for is the long-term objective of our collaborative research program. These data provide an important first step towards this goal.

78046

The Kansas City Quality \& Value Innovation Consortium (KC QVIC): Leveraging Team Science, Translational Research and Training to Improve the Value of Healthcare in the Community

Stacy Farr ${ }^{1}$, Jon Spertus ${ }^{1}$, James Stowe ${ }^{2}$, Holly Hagle ${ }^{3}$ and Jacque Sawyer

${ }^{1}$ Saint Luke's Health System and University of Missouri-Kansas City; ${ }^{2}$ Mid America Regional Council (MARC); ${ }^{3}$ University of MissouriKansas City

ABSTRACT IMPACT: Implementing a team science approach with broad engagement from academic researchers, healthcare payers, providers, patients, and community-based organizations is complex, yet critical to implementing evidence into real world settings. OBJECTIVES/GOALS: 1. Participants will be able to deploy novel strategies for creating and training a regional multi-stakeholder consortium to improve the quality and value of healthcare.

2. Participants will be to examine ways in which team science provides holistic sustainable strategies to improve care and outcomes in real-world settings. METHODS/STUDY POPULATION: The Quality \& Value Innovation Consortium (QVIC) has created a network of hospitals and other stakeholders (providers, payers, purchasers, patients, community-based organizations, and researchers) to collaborate and innovate on healthcare delivery. This initiative began with a team of a physician researcher, a health services researcher, and a nurse researcher first identifying healthcare systems' priorities through individual meetings with leadership from 14 regional hospitals. Concurrently, meetings were held with other stakeholders. These interviews identified 32 key quality improvement topics.

Focus groups and surveys reduced these to 11 topics that were then selected for community forums. Through a mixed methods approach, two priority topics were selected for regional implementation. RESULTS/ANTICIPATED RESULTS: The QVIC meetings have prioritized two topics and highlighted novel information sharing across entities, and strategies to address the social determinants of health. The QVIC efforts have been recognized as a community asset for helping build collaboration and partnerships among diverse stakeholders. Ultimately, two regional initiatives, opioid management, and transitions in heart failure care were selected for implementation. Both of these initiatives aim to reduce readmissions by addressing social determinants of health. Implementation strategies and evaluation metrics are being customized for pragmatic integration within each system, utilizing a collaborative team science approach. DISCUSSION/SIGNIFICANCE OF FINDINGS: While the entire country is grappling with the challenge of improving the quality of care, while lowering its costs, Kansas City has modeled a unique culture and strategy for achieving this goal, important for learning health systems and communities.

87842

\section{The Effects of Head Impact Exposure on Changes in Neurocognitive and Oculomotor Function Across a High School American Football Season: A Pilot Study}

Megan E. Huibregtse, Jonathan T. Macy, Jesse A. Steinfeldt and Keisuke Kawata

School of Public Health-Bloomington, Indiana University

ABSTRACT IMPACT: This work reveals the influence of a season of American football-related head impact exposure on two functional outcome measures in a cohort of adolescent boys, shedding light on the chronic effects of 'subconcussive head impacts.' OBJECTIVES/GOALS: To examine the influence of a season of exposure to head impacts in American football on changes in neurocognitive and oculomotor function in adolescent male athletes. METHODS/STUDY POPULATION: Participants were recruited from a local high school: the football group $(\mathrm{FB} ; \mathrm{n}=26)$ was instrumented with sensor-installed mouthguards to track impact exposure during games and practices, and members of the men's cross-country team were recruited to the control group $(\mathrm{CON} ; \mathrm{n}=9)$. All participants were administered Immediate Post-concussion Assessment and Cognitive Testing (ImPACT) and were assessed for near point of convergence (NPC) at pre- and post-season. Linear models will be fit for changes in the five ImPACT composite scores and NPC values, with group and one of the head impact variables as predictors for each model. In a secondary within-group analysis, correlation coefficients will be calculated for the relationships between the head impact variables and the functional change scores for the FB group. RESULTS/ANTICIPATED RESULTS: The two groups did not differ significantly on age or number of previous concussions; the CON group had significantly lower BMI. Group assignment was significantly associated with change in NPC ( $p<0.05$ for all three models); no significant associations were observed for any of the head impact variables with change in NPC. Group and each of the head impact variables (total impacts, sum of peak linear acceleration [PLA], and sum of peak rotational acceleration [PRA]) were not significantly associated with change in any of the five ImPACT composite scores. Change in visual memory composite score was negatively correlated with total impacts $(r=-0.37, p=0.034)$ and sum of PRA $(r=-0.36$, $\mathrm{p}=0.040)$. DISCUSSION/SIGNIFICANCE OF FINDINGS: Significant, albeit relatively weak, correlations between change in visual memory composite score and two head impact kinematic variables, coupled with significant increases in NPC in the FB group compared to the CON group, suggest that a season of exposure to football-related head impacts has the potential to elicit minor functional impairments. 